\title{
EFEITO DO PLANO NUTRICIONAL SOBRE AS MEDIDAS BIOMÉTRICAS DE CORDEIRAS DURANTE A FASE DE CRESCIMENTO
}

Ariane Dantas ${ }^{1}$, Rogério Antônio Oliveira ${ }^{2}$, Maria Fernanda Correia Silva Carrega ${ }^{1}$, Felipe Rydygier de Ruediger $^{1}$, André Michel de Castilho ${ }^{3}$, Eunice Oba ${ }^{1}$

${ }^{1}$ Universidade Estadual Paulista - UNESP, Departamento de Reprodução Animal e Radiologia Veterinária, Faculdade de Medicina Veterinária e Zootecnia, Botucatu, SP. ${ }^{2}$ Universidade Estadual Paulista - UNESP, Departamento de Bioestatística do Instituto de Biociência, Botucatu,SP. ${ }^{3}$ Universidade Estadual Paulista - UNESP, Departamento de Melhoramento e Nutrição Animal, Botucatu, SP. E-mail: dantas.vet@gmail.com

\section{RESUMO}

O objetivo deste estudo foi avaliar a influência e relação de duas taxas de crescimento com o desenvolvimento corporal de cordeiras, mediante o monitoramento das medidas corporais. Foram avaliadas 20 cordeiras da raça Bergamácia mantidas confinadas e recebendo uma dieta formulada para obtenção ganho de peso médio diário (GPMD) de 180 gramas (tratamento Baixo) e 300 gramas (tratamento Alto). A cada 14 dias os animais foram pesados, determinado o escore de condição corporal (ECC), a altura, os perímetros torácico, do pescoço e da canela. Para avaliação dos dados utilizou-se análise de variância e correlação de Spearman com diferença significativa quando $\mathrm{P}<0,05$. As cordeiras do tratamento Alto foram as que apresentaram maior GPMD, ECC e medidas biométricas, além disso, nos dois tratamentos, o padrão biométrico correlacionou-se positivamente entre si e com o peso corporal. Portanto, a diferenciação alimentar teve efeito direto no desenvolvimento de cordeiras em crescimento.

Palavras-chave: manejo alimentar; ovino; desenvolvimento corporal.

\section{EFFECT OF NUTRITION PLAN ON BIOMETRIC MEASUREMENTS OF LAMBS DURING THE GROWTH PHASE}

\begin{abstract}
The aim of this study was to evaluate the influence and relationship of two growth rates with body development lambs, by monitoring the body measurements. We evaluated 20 Bergamasca lambs kept confined and offered a diet formulated to obtain an average daily gain weight (ADG) of 180 grams (treatment Low) and 300 grams (treatment High). Every 14 days, the animals were weighed, given the body condition score (BCS), height, chest girth, neck and shin. To evaluate the data we used analysis of variance and correlation with Spearman significant difference when $\mathrm{P}<0.05$. Lambs of treatment High showed the highest $A D G, B C S$ and biometric measurements, in addition, in both treatments, the biometric pattern correlated positively with each other and the body weight. Therefore, food differentiation had a direct effect on the development of growing lambs.
\end{abstract}

Keywords: feed management; sheep; body development.

\section{INTRODUÇÃO}

Em qualquer sistema de produção animal, o manejo nutricional adequado de fêmeas para reposição é um fator muito importante a ser considerado por influenciar diretamente a idade à puberdade e à primeira cobrição, o peso ao parto e a posterior produção de leite (DANTAS, 2013).
A alimentação adequada associada à determinação do peso e da manutenção da condição corporal torna-se essencial para o acompanhamento do crescimento e para assegurar bom desempenho produtivo $e$ reprodutivo de fêmeas ruminantes (CEZAR; SOUZA, 2006; ROBINSON et al., 2006).

Desta forma, é de extrema importância o conhecimento das medidas corporais e as 
correlações dessas com o peso corporal, pois permitem comparações entre animais e definições de grupamentos genéticos, sistemas de alimentação, além de serem estratégias comumente usadas em programas de seleção e de produção de ovinos (FILHO et al., 2010).

Entretanto, valores de referência para cordeiras confinadas e em fase crescimento, bem como para as da raça Bergamácia são escassos, todavia, são fundamentais para o fortalecimento da atividade leiteira.

O objetivo do presente experimento foi avaliar a influência de duas taxas de crescimento sobre o desenvolvimento corporal de cordeiras confinadas, mediante o monitoramento das medidas corporais, durante a fase de crescimento.

\section{METODOLOGIA}

$O$ experimento foi realizado na Universidade Estadual Paulista (UNESP), Faculdade de Medicina Veterinária e Zootecnia, Campus de Botucatu/SP, no período de julho de 2011 a abril de 2012, nas instalações da Unidade de Pesquisa em Produção de Leite Ovino e aprovado pela Comissão de Ética no Uso de Animais (CEUA) da Faculdade de Medicina Veterinária e Zootecnia, UNESP, Botucatu-SP, protocolo número 159/2010.

Foram utilizadas 20 cordeiras da raça Bergamácia com idade inicial de 90 dias, oriundas de partos simples, divididas aleatoriamente em dois tratamentos: um para obtenção de baixo ganho de peso médio diário (180 g) e o outro para alto ganho de peso médio diário (300 g). Foram dispostos dois animais do mesmo tratamento por baia até completarem 1 ano de idade. $\mathrm{O}$ tamanho amostral baseou-se no número total de cordeiras oriundas de partos simples, visto que animais de nascimento simples apresentam maior potencial de crescimento do que os de nascimento duplo (BARROS et al., 2005). Assim, de um rebanho de 48 ovelhas gestantes, 20 fêmeas tiveram partos simples.

As dietas foram formuladas de acordo com o National Research Council - NRC (2007), sendo os ingredientes utilizados nas dietas apresentados na Tabela 1. Para as cordeiras do tratamento Alto foi adotado um plano de adaptação à dieta, cuja finalidade foi evitar distúrbios metabólicos e consistiu no fornecimento de feno: concentrado na proporção de $40: 60 \%$ nos 7 primeiros dias; de $30: 70 \%$ nos 14 dias e de $20: 80 \%$ nos 7 últimos dias, totalizando 28 dias de adaptação.

Optou-se em adotar uma dieta com alta quantidade de concentrado (Tratamento 2), devido ao fato de que muitos produtores alimentam suas cordeiras sem distinguir as que serão destinadas ao mercado, daquelas que serão utilizadas para reposição, permitindo acesso livre à dietas elaboradas com a finalidade de promover rápido ganho de peso (VILLENEUVE et al., 2010), sem, contudo, qualquer comprovação científica sobre os possíveis efeitos que esse tipo de manejo alimentar pode ter em cordeiras com aptidão leiteira, com as da raça Bergamácia.

Os animais foram mantidos confinados durante toda a fase experimental, em baias cobertas ( $3,50 \mathrm{~m} \times 1,75 \mathrm{~m})$, com piso de concreto, seguindo as recomendações da Embrapa (2009) quanto as dimensões e densidade animal para ovinos criados em sistema intensivo. Cada baia possuía cocho para fornecimento de ração $(0,95$ $\mathrm{m} \times 0,35 \mathrm{~m}$ ) e bebedouro com bóia. 
Tabela 1. Proporção feno:concentrado, composição alimentar e nutricional das dietas experimentais

\begin{tabular}{lrr}
\hline Ingredientes (g/kg de MS) & Baixo \\
\hline Feno Cynodon dactylon & 624,50 & 202,00 \\
Concentrado $^{\prime}$ & 375,50 & 798,00 \\
Milho moído & 235,80 & 572,30 \\
Farelo de Soja $^{\text {Calcário calcítico }}$ & 118,40 & 202,00 \\
Sal mineral $^{1}$ & 6,10 & 10,40 \\
Monensina sódica $_{\text {Nutrientes (g/kg de MS) }}$ & 12,10 & 10,40 \\
\hline PB & 0,030 & 0,030 \\
NDT & & \\
EE & 11,34 & 16,04 \\
MM & 65,12 & 78,20 \\
FB & 1,70 & 3,44 \\
FDN & 5,46 & 4,53 \\
FDA & 24,18 & 9,70 \\
\hline COmposça & 56,39 & 28,00 \\
\hline
\end{tabular}

${ }^{1}$ Composição do Sal Mineral (kg do produto) $155 \mathrm{~g} \mathrm{Ca}$, 65g P, $6 \mathrm{~g} \mathrm{mg}, 12 \mathrm{~g} \mathrm{~S}, 115 \mathrm{~g} \mathrm{Na}, 27 \mathrm{mg} \mathrm{Se}, 100 \mathrm{mg} \mathrm{Cu}, 1000 \mathrm{mg} \mathrm{Fe}$, $6000 \mathrm{mg} \mathrm{Zn}, 1400 \mathrm{mg} \mathrm{Mn}, 175 \mathrm{mg} \mathrm{l}, 175 \mathrm{mg} \mathrm{Co}, 650 \mathrm{mg} \mathrm{Fl}$.

Baixo: Dieta formulada para obtenção de ganho médio diário de 180 gramas; Alto: dieta formulada para obtenção de ganho médio diário de 300 gramas.

Os animais foram alimentados duas vezes ao dia, às $8 \mathrm{~h} 00$ e às $15 \mathrm{~h} 00$, com acesso ad libitum às dietas e à água. A cada 14 dias as cordeiras foram pesadas e o peso corporal (PC) expresso em quilograma $(\mathrm{kg})$. O ganho peso médio diário (GPMD) foi calculado considerando o ganho das cordeiras durante o período dividido pelo número de dias entre as pesagens (14 dias).

Mensurou-se também o escore de condição corporal (ECC) conforme descrito por Sañudo e Sierra (1986) e com auxílio de fita métrica, foram aferidas as medidas biométricas, sendo essas: altura da cernelha- AC (distância entre a região da cernelha e a extremidade distal do membro anterior), comprimento corporal- CC (distância entre a articulação cérvico-torácica e a base da cauda na primeira articulação intercoccígea), perímetro torácico- PT (tomandose como base o esterno e a cernelha) de acordo com Searle et al. (1989), além dos perímetros do pescoço- PP e da canela- PCA.

$O$ experimento foi conduzido em delineamento inteiramente casualizado e os dados das características de GPMD e total e ECC foram submetidos à análise de variância com uso do software estatístico SAS versão 9.3 (SAS INSTITUTE INC., CARY, NC, EUA). Para estudar a possível relação linear entre os valores das medidas corporais e dessas com o PC, durante o período experimental, foram calculadas correlações não-paramétricas de Spearman, pois as distribuições das medidas biométricas foram assimétricas. O coeficiente de correlação foi considerado como nulo $(r=0)$, baixo $(0<r \leq 0,30)$, moderado $(r=0,30<r \leq 0,70)$ e forte $(0,70<r \leq$ 1), segundo Levine et al. (2012).

\section{RESULTADOS}

Houve diferença significativa para as variáveis estudas, sendo as cordeiras do tratamento Alto as que apresentaram os maiores valores (Tabela 2), apresentando aumento no ganho corporal de $42,22 \mathrm{~kg}$ em relação ao peso inicial, comparado a $51,27 \mathrm{~kg}$ do tratamento Baixa (Figura 1). 
Tabela 2. Médias de peso, ganho de peso médio diário e escore de condição corporal das cordeiras submetidas aos tratamentos Baixo e Alto

\begin{tabular}{lrr}
\hline & Baixo $\pm \mathrm{DP}$ & Alto $\pm \mathrm{DP}$ \\
\hline Peso corporal inicial $(\mathrm{kg})$ & $14,95 \pm 1,98$ & $13,90 \pm 1,53$ \\
**Peso corporal final $(\mathrm{kg})$ & $57,17 \pm 4,09$ & $65,17 \pm 3,86$ \\
**Ganho de peso médio diário $(\mathrm{g})$ & $159,00 \pm 0,07$ & $193,00 \pm 0,05$ \\
**Escore de condição corporal inicial & $1,42 \pm 0,51$ & $1,74 \pm 0,76$ \\
**Escore de condição corporal final & $2,76 \pm 0,24$ & $3,12 \pm 0,22$ \\
\hline
\end{tabular}

Baixo: Dieta formulada para obtenção de ganho médio diário de 180 gramas; Alto: dieta formulada para obtenção de ganho médio diário de 300 gramas. DP: Desvio Padrão. ${ }^{* *}(\mathrm{P}<0,05)$

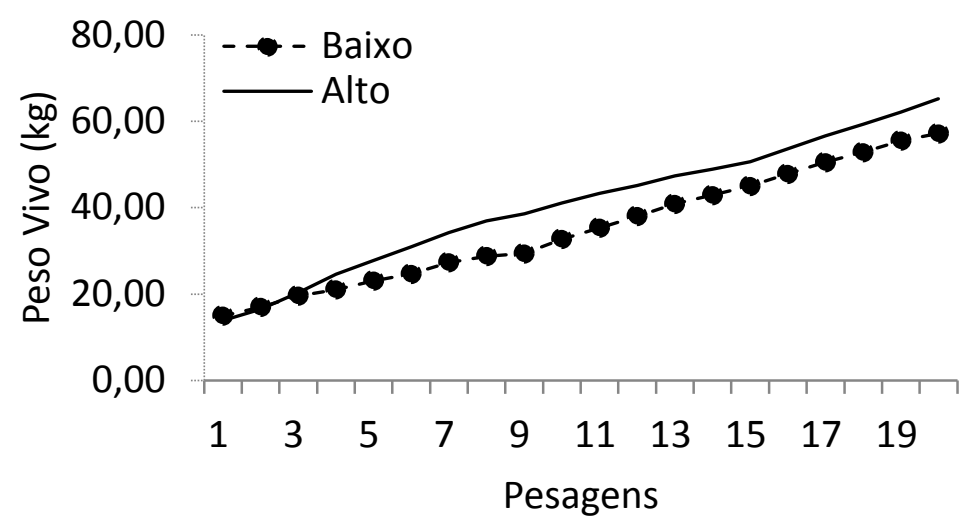

Figura 1. Média do peso vivo das cordeiras dos tratamentos Baixo e Alto durante o período experimental

Foi observado também diferenças significativas $(P<0,05)$ entre os tratamentos para todas as médias dos valores das medidas biométricas, sendo as cordeiras do tratamento
Alto as que apresentaram os maiores valores (Tabela 3).

Tabela 3. Médias dos valores das medidas biométricas $(\mathrm{cm})$ das cordeiras submetidas aos tratamentos Baixo e Alto

Medidas (cm)

$* *$ Comprimento Corporal (CC)

**Altura da Cernelha (AC)

**Perímetro Torácico (PT)

**Perímetro do Pescoço (PP)

**Perímetro da canela (PCA)

\begin{tabular}{rr} 
Baixo \pm DP & Alto \pm DP \\
\hline $84,73 \pm 11,08$ & $87,91 \pm 11,99$ \\
$66,53 \pm 6,53$ & $69,41 \pm 7,72$ \\
$78,44 \pm 13,77$ & $81,15 \pm 13,76$ \\
$30,17 \pm 3,43$ & $31,52 \pm 3,45$ \\
$10,89 \pm 1,85$ & $11,57 \pm 1,97$
\end{tabular}

Baixo: dieta formulada para obtenção de ganho de peso médio diário de 180 gramas; Alto: dieta formulada para obtenção de ganho de peso médio diário de 300 gramas.

DP: Desvio Padrão. ** $(\mathrm{P}<0,05)$

Em ambos os tratamentos, as medidas biométricas correlacionaram-se forte e positivamente entre si, bem como com o PC, sendo a relação entre o PT e o PC a de maior magnitude (Tabela 4). 
Tabela 4. Correlações entre as medidas biométricas, altura da cernelha (AC), comprimento corporal (CC), perímetro torácico (PT), perímetro do pescoço (PP), perímetro da canela (PCA) e peso corporal (PC), das cordeiras Bergamácia submetidas aos tratamentos Baixo e Alto

\begin{tabular}{|c|c|c|c|c|c|c|c|c|c|c|c|}
\hline & \multicolumn{5}{|c|}{ Baixo } & \multicolumn{6}{|c|}{ Alto } \\
\hline & $A C$ & CC & PT & PP & PCA & & $A C$ & CC & PT & PP & PCA \\
\hline \multirow{2}{*}{$\mathrm{CC}$} & 0,96 & & & & & \multirow{2}{*}{$\mathrm{CC}$} & 0,94 & & & & \\
\hline & $<0,0001$ & & & & & & $<0,0001$ & & & & \\
\hline \multirow{2}{*}{ PT } & 0,96 & 0,97 & & & & \multirow{2}{*}{ PT } & 0,93 & 0,95 & & & \\
\hline & $<0,0001$ & $<0,0001$ & & & & & $<0,0001$ & $<0,0001$ & & & \\
\hline \multirow{2}{*}{ PP } & 0,92 & 0,94 & 0,96 & & & \multirow{2}{*}{ PP } & 0,91 & 0,92 & 0,95 & & \\
\hline & $<0,0001$ & $<0,0001$ & $<0,0001$ & & & & $<0,0001$ & $<0,0001$ & $<0,0001$ & & \\
\hline \multirow{2}{*}{ PCA } & 0,94 & 0,95 & 0,96 & 0,94 & & \multirow{2}{*}{ PCA } & 0,91 & 0,94 & 0,96 & 0,93 & \\
\hline & $<0,0001$ & $<0,0001$ & $<0,0001$ & $<0,0001$ & & & $<0,0001$ & $<0,0001$ & $<0,0001$ & $<0,0001$ & \\
\hline \multirow{2}{*}{ PC } & 0,96 & 0,97 & 0,99 & 0,95 & 0,96 & \multirow{2}{*}{ PC } & 0,93 & 0,96 & 0,98 & 0,96 & 0,95 \\
\hline & $<0,0001$ & $<0,0001$ & $<0,0001$ & $<0,0001$ & $<0,0001$ & & $<0,0001$ & $<0,0001$ & $<0,0001$ & $<0,0001$ & $<0,0001$ \\
\hline
\end{tabular}

Baixo: dieta formulada para obtenção de ganho de peso médio diário de 180 gramas; Alto: dieta formulada para obtenção de ganho de peso médio diário de 300 gramas. ${ }^{* *}(\mathrm{P}<0,01)$

\section{DISCUSSÃO}

As cordeiras do tratamento Alto apresentaram melhor desempenho corporal durante todo o estudo devido o fornecimento de um nível nutricional elevado, visto que dietas com maior concentração energética proporcionam maior disponibilidade de nutrientes para o crescimento corporal, permitindo maior ganho de peso (IGNÁCIO et al., 2003). Entretanto, o GPMD das cordeiras desse tratamento, foi baixo. Segundo Mertens (1994) o aumento na proporção de concentrado nas dietas resulta em aumento do desempenho corporal até que as exigências de energia sejam alcançadas. Assim, a exigência energética das cordeiras do tratamento Alto pode ter sido alcançada com níveis menores de ingestão da dieta, resultando em baixo GPMD.

Os parâmetros analisados refletiram as diferenças esperadas entre os dois regimes alimentares, estando os valores encontrados em conformidade com Haddad e Husein (2004) em trabalho realizado com cordeiros da raça leiteira Awassi em fase de crescimento, submetidos a dietas com $40 \%$ e $85 \%$ de concentrado, onde maior ganho de peso, foi observado naqueles que receberam dieta com maior proporção de concentrado.

As cordeiras do tratamento Alto apresentaram melhor desempenho, correspondendo ao alto plano nutricional, evidenciando seu potencial para ganho de peso. Segundo Susin (1996), desde que não haja deposição excessiva de gordura corporal, o alto nível nutricional do nascimento ao primeiro parto, é benéfico.

Os resultados encontrados para as medidas biométricas mostraram que ambos os tratamentos foram eficientes para o desenvolvimento corporal das cordeiras, sendo também semelhantes aos obtidos por Pinheiro e Jorge (2010), ao estudarem a morfometria de ovelhas de descarte da raça Santa Inês.

As correlações entre as medidas biométricas e a relação dessas com PC foram positivas, bem como de forte magnitude, dessa forma, o aumento de uma variável ocorreu estreitamente associada ao incremento da outra, sendo o PT melhor relacionado ao PC. Estes dados são importantes, pois segundo Santana et al. (2001), estão relacionados com a capacidade digestiva e respiratória; e consequentemente com o desenvolvimento dos animais.

Corroborando Memória et al. (2005), que afirmaram que as medidas biométricas independem da idade do animal, sendo a altura da cernelha e o perímetro torácico as mais correlacionadas com o PC. Estudos em ovinos da raça Morada Nova (URBANO et al., 2006) e Santa Inês (MAGALHÃES et al., 2006) ambos com cordeiras em fase de crescimento, encontraram o PT como a medida mais correlacionada ao peso, corroborando os dados encontrados no presente estudo.

\section{CONCLUSÃO}

As dietas do tratamento Baixo e Alto resultaram em bom desenvolvimento corporal 
em ambos os grupos, permitindo a obtenção de excelentes medidas corporais. Dessa forma, a alimentação de cordeiras com um plano moderado de nutrição pode ser considerada como uma estratégia de otimização do manejo nutricional de cordeiras confinadas durante a fase de crescimento.

\section{REFERÊNCIAS}

BARROS, N.N.; VASCONCELOS, V.R.; WANDER, A.E.; ARAÚJO, M.R.A. Eficiência bioeconômica de cordeiros F1 Dorper x Santa Inês para produção de carne. Pesquisa Agropecuária Brasileira, v.40, p.825-831, 2005.

http://dx.doi.org/10.1590/S0100-

204X2005000800014

CEZAR, M.F.; SOUSA, W.H. Avaliação e utilização da condição corporal como ferramenta de melhoria da reprodução de ovinos e caprinos de corte. In: REUNIÃO ANUAL DA SOCIEDADE BRASILEIRA DE ZOOTECNIA, 43., 2006, João Pessoa, PB. Simpósios. Anais... João Pessoa: SBZ, 2006. p.649-678.

DANTAS, A. Idade à puberdade e desenvolvimento da glândula mamária de cordeiras sob baixa e alta velocidades de crescimento. 2013. 78 f. Dissertação (Mestrado em Zootecnia) - Faculdade de Medicina Veterinária e Zootecnia, Universidade Estadual Paulista, Botucatu, 2013.

EMBRAPA. Estresse pode diminuir produtividade animal. 2009. Disponível em: <http://embrapa.br>. Acesso em: 09 ago. 2016. FILHO, L. F. C. C; REGO, F. C. A; JUNIOR, F. A. B; STERZA, F. A. M; OKANO, W; TRAPP, S. M. Predição do peso corporal a partir de mensurações corporais em ovinos texel. Arquivos de Ciências Veterinárias e Zoologia da UNIPAR, v.13, n.1, p.5-7, jan./jun. 2010.

HADDAD, S.G.; HUSEIN, M.Q. Effect of dietary energy density on growth performance and slaughtering characteristics of fattening Awassi lambs. Livestock Production Science, v.87, p.171177, 2004. http://doi:10.1016/j.livprodsci.2003.09.018 IGNÁCIO, R.I.; CLARK, J.H. Usefulness of ionophores for lactating dairy cows: a review. Animal Feed Science and Technology, v.106, p.39-57, 2003.

http://doi:10.1016/S0377-8401(03)00065-8

LEVINE, D. M.; BERENSON, M. L.; STEPHAN, D. Estatística: teoria e aplicações - Usando Microsoft Excel. 6. ed. Rio de Janeiro: LTC, 2012.
MAGALHÃES, A.F.B.; GOMES, G.L.; CARVALHO, R.S.; NASCIMENTO JÚNIOR, P. S. DO; SIMEÃO, R. S. F.; COSTA, H. H. A.; FONTENELE, R. M.; ROGÉRIO, M. C. P.; MARTINS, G. A. Determinação do peso vivo de ovinos Santa Inês e SRD a partir de equações de regressão múltipla. In: ZOOTEC, REUNIÃO ANUAL. Anais... Pernambuco: Zootec, 2006. CD-ROM.

MEMÓRIA, H.Q.; DO REGO, J.P.A.; CATUNDA, A.C.V.; GUIMARÃES, A.N.C.; ROGÉRIO, M.C.P.; MARTINS, G.A. (2005) Correlação entre peso e medidas corporais em ovinos machos de diferentes idades. In: ZOOTEC, REUNIÃO ANNUAL.. Anais... Pernambuco: Zootec, 2005. CD-ROM.

MERTENS, O. Regulation of forage intake. In: NATIONAL CONFERENCE ON FORAGE QUALITY, EVALUATION, AND UTILIZATION. Anais... Lincoln: University of Nebraska, 1994. p.450-493.

NATIONAL RESEARCH COUNCIL - NRC. Nutrient requeriments of sheep. 6. ed. Washington: National Academy Press, 2007.

PINHEIRO, R. S. B.; JORGE, A. M. Medidas biométricas obtidas in vivo e na carcaça de ovelhas de descarte em diferentes estágios fisiológicos. Revista Brasileira de Zootecnia, v.39, n.2, p.440-445, 2010. http://dx.doi.org/10.1590/S1516-

35982010000200030.

ROBINSON, J.J.; ASHWORTH, C.J.; ROOKE, J.A.; MITCHELL, L. M.; MCEVOY, T. G. Nutrition and fertility in ruminant livestock. Animal Feed Science and Technology, v.126, p.259-276, 2006. http://dx.doi.org/10.1016/j.anifeedsci.2005.08.0 06

SANTANA, A.F.; COSTA, G.B.; FONSECA, L.S. Correlações entre peso e medidas corporais em ovinos jovens da raça Santa Inês. Revista Brasileira de Saúde e Produção Animal do Brasil, v.1, n.3, p.74-77, 2001.

SAÑUDO, C.; SIERRA, I. Calidad de la canal en la especie ovina. Ovino, n.1, p.127-153, 1986.

SEARLE, T.W.; GRAHAM, M.; DONNELLY, J.B. Change of skeletal dimensions during growth in sheep: the effect of nutrition. Journal of Agricultural Science, v.112, n.3, p.321-327, 1989. http://dx.doi.org/10.1017/S0021859600085774 SUSIN, I. Nutrição de Ovinos. 1. ed. Jaboticabal: Funep, 1996.

URBANO, S.A., CÂNDIDO, E.P.; DE LIMA, C.A.C.; CARVALHO, M. D. F.; ARAÚJO, P. M.; GODEIRO, J. R. G.; FONSECA, F. C. E.; CAVALCANTI, F. A. L. Uso da barimetria para estimar o peso corporal de 
ovinos da raça morada nova. In: ZOOTEC 2006.

Anais... Pernambuco: 2006. CD-ROM.

VILLENEUVE, L.; CINQ-MARS, D., LACASSE, P. Effects of restricted feeding of prepubertal ewe lambs on growth performance and mammary gland development. Animal, Clermont-Theix, v.4, p.944-950, 2010.

10.1017/S1751731110000170.

Recebido para publicação em 16/08/2016

Revisado em 19/08/2016

Aceito em 22/08/2016 